\title{
APPROXIMATIONS TO THE DISTRIBUTION OF A COMBINATION OF THE WILCOXON AND MOOD STATISTICS: A NUMERICAL COMPARISON
}

\author{
Hidetoshi Murakami*
}

\begin{abstract}
On testing hypotheses in two-sample problems, the Lepage-type statistic is often used for testing the location and scale parameters. Various Lepage-type statistics have been proposed and discussed by many authors over the course of many years. One of the most famous and powerful Lepage-type statistics is a combination of the Wilcoxon and Mood statistics, namely $T$. Deriving the exact critical value of the $T$ statistic is difficult when the sample sizes are increased. In that situation, an approximation to the distribution function of a test statistic is extremely important in statistics. The gamma approximation and the saddlepoint approximations are used to evaluate in upper tail probability for the $T$ statistic under finite sample sizes. The accuracy of various approximations to the exact probability of the $T$ statistic is investigated.
\end{abstract}

\section{Introduction}

Testing hypotheses is one of the most important problems in performing nonparametric statistics. On testing hypotheses in two-sample problems, we have to test the location and scale parameters at the same time in many cases. If the scale parameters change, the test statistic for the location parameter is not useful. Similarly, if the location parameters change, the test statistic for the scale parameter is not useful. To resolve the dilemma, Lepage (1971) developed the test statistic that combined the Wilcoxon statistic (Gibbons and Chakraborti, 2003) and the Ansari-Bradley statistic (1960). After the Lepage statistic was proposed, various Lepage-type statistics have been proposed and discussed over the course of many years, e.g. Büning and Thaewald (2000), Neuhäuser (2000), Büning (2002), Murakami (2007). One of the most famous and powerful Lepage-type statistics is a combination of the Wilcoxon and Mood (1954) statistics, namely $T$, proposed by Pettitt (1976). To calculate the exact critical value of the test statistic is an important problem in testing hypotheses. However, it is difficult to obtain the exact critical value when the sample sizes are increased. Under these circumstances, we must estimate the exact critical value with an approximation method. Hence, considering approximations for evaluating the density or distribution function of the test statistic remains one of the most important topics in statistics. For the approximation presented in this paper, we used a gamma approximation and a saddlepoint approximation proposed by Daniels $(1954,1987)$ and specifically of the type developed by Lugannani and Rice (1980). The saddlepoint approximations have the advantage in the tails of the distribution. Saddlepoint approximations have been used with great success by many authors, and excellent discussions of their applications to a range

*Department of Mathematics, National Defense Academy, 1-10-20 Hashirimizu, Yokosuka-shi, Kanagawa 239-8686, Japan E-mail: murakami@indsys.chuo-u.ac.jp

Key words: Cumulant generating function; Gamma approximation; Nonparametric test; Numerical comparison; Saddlepoint approximation 
of distributional problems are provided by Reid (1988), Jensen (1995), Goutis and Casella (1999), Huzurbazar (1999), Kolassa (2006) and Butler (2007). The saddlepoint approximation can be obtained for any statistic that admits a cumulant generating function (CGF). When the CGF is unknown or difficult to derive, Easton and Ronchetti (1986) and Wang (1992) proposed obtaining approximations to the CGF by using expansion.

For conducting a distribution-free test, Giles (2001) and Chen and Giles (2008) treated the saddlepoint approximations to the limiting distribution of the Anderson-Darling statistic $(1952,1954)$ and found that the saddlepoint approximations were better than the approximation of Sinclair and Spurr (1998). In addition, Murakami (2009) indicated that the saddlepoint approximation was better than the approximation of Sinclair, Spurr and Ahmad (1990) to the limiting distribution of the modified Anderson-Darling statistic (1990).

In nonparametric statistics, researchers are very interested in considering approximations under finite sample sizes. Froda and van Eeden (2000) proposed a uniform saddlepoint expansion to the null distribution of the Wilcoxon-Mann-Whitney test. Additionally, Bean, Froda and van Eeden (2004) compared a saddlepoint approximation of the Wilcoxon-MannWhitney test with that of Edgeworth, and determined normal and uniform approximations under finite sample sizes. Recently, Murakami and Kamakura (2009) considered a saddlepoint approximation to the distribution of the Jonckheere-Terpstra test (Gibbons and Chakraborti, 2003) with a continuity correction. In addition, Murakami (2010) investigated the accuracy of a saddlepoint approximation with a continuity correction to the distribution of a test statistic for a competing risks model. Most of the previous works were focused on the statistics for location or scale changes. Once again, we often require testing the location and scale parameters at the same time. In this paper, we focus on a combination of the Wilcoxon and Mood statistics. In Section 2, we introduce the $T$ statistic and list some exact critical values of the $T$ statistic for small sample sizes. In addition, we give the explicit first four cumulants of the $T$ statistic. Note that we might not evaluate the exact critical value of the $T$ statistic when the sample sizes are increased. In that case, by using the cumulants, we suggest the gamma approximation. Since it is also difficult to derive the CGF of the $T$ statistic, we consider the approximation to the CGF of the $T$ statistic. In Section 3, we report on the saddlepoint approximations to the distribution of the $T$ statistic. In Section 4 , we compare the accuracy of the gamma approximation, normal-based and nonnormal-based saddlepoint approximations to the distribution of the $T$ statistic under finite sample sizes. Finally, we conclude this paper in Section 5.

\section{A combination of the Wilcoxon and Mood statistics}

In this section, we introduce a modification of the Lepage statistic. Let $X=\left(X_{1}, \ldots, X_{n}\right)$ and $Y=\left(Y_{1}, \ldots, Y_{m}\right)$ be two random samples of size $n$ and $m$ independent observations, each of which had a continuous distribution described as $F_{1}$ and $F_{2}$, respectively. Pettitt (1976) first described the use of the Mood statistic in a Lepage-type test as follows:

$$
T=\left(\frac{W-\mathrm{E}(W)}{\sqrt{\operatorname{var}(W)}}\right)^{2}+\left(\frac{M-\mathrm{E}(M)}{\sqrt{\operatorname{var}(M)}}\right)^{2},
$$

where

$$
W=\sum_{i=1}^{N} i V_{i} \quad \text { and } \quad M=\sum_{i=1}^{N}\left(i-\frac{N+1}{2}\right)^{2} V_{i}
$$


Let $V_{i}=1$ if the $i$ th smallest of the $N=n+m$ observations is from $X$ and $V_{i}=0$ otherwise. $\mathrm{E}(\cdot)$ and $\operatorname{var}(\cdot)$ denote the mean and variance under the null hypothesis. The limiting distribution of the $T$ statistic is known to be a chi-square distribution with two degrees of freedom (Pettitt, 1976). Herein, we give the exact first four cumulants of the $T$ statistic as follows:

$$
\begin{aligned}
\kappa_{1} & =2, \\
\kappa_{2} & =\frac{4\left(A_{1}+A_{2}\right)}{35 n m(N-2)^{2}(N+1)(N+2)}=\frac{4 N^{4}}{(N-2)^{2}(N+1)(N+2)}+O\left(N^{-1}\right), \\
\kappa_{3} & =\frac{16\left(A_{3}+A_{4}+A_{5}+A_{6}\right)}{35035 n^{2} m^{2}(N-2)^{3}(N+1)^{2}(N+2)^{2}}=\frac{16 N^{7}}{(N-2)^{3}(N+1)^{2}(N+2)^{2}}+O\left(N^{-1}\right), \\
\kappa_{4} & =\frac{96\left(A_{7}+A_{8}+A_{9}+A_{10}+A_{11}+A_{12}\right)}{2977975 n^{3} m^{3}(N-2)^{4}(N+1)^{3}(N+2)^{3}}=\frac{96 N^{10}}{(N-2)^{4}(N+1)^{3}(N+2)^{3}}+O\left(N^{-1}\right) .
\end{aligned}
$$

See Appendix for $A_{1}$ to $A_{12}$. Additionally, we list the exact critical value of the $T$ statistic in Table 1.

Table 1: Exact critical value of the $T$ statistic

\begin{tabular}{cccc|cccc}
\hline$n$ & $m$ & $\operatorname{Pr}(T \geq t)$ & $t$ & $n$ & $m$ & $\operatorname{Pr}(T \geq t)$ & $t$ \\
\hline 7 & 7 & 0.0099 & 7.25000 & 10 & 5 & 0.0100 & 7.69484 \\
& & 0.0245 & 6.40408 & & & 0.0250 & 6.43484 \\
& & 0.0490 & 5.56020 & & & 0.0496 & 5.34045 \\
\multirow{4}{*}{10} & \multirow{2}{*}{10} & 0.0997 & 4.46531 & & & 0.0999 & 4.35955 \\
& & 0.0100 & 7.95671 & 30 & 5 & 0.0100 & 8.89485 \\
& & 0.0250 & 6.72156 & & & 0.0250 & 6.89588 \\
& & 0.0499 & 5.69229 & & & 0.0500 & 5.48300 \\
13 & 0.0996 & 4.53541 & & & 0.0999 & 4.27949 \\
& \multirow{3}{*}{13} & 0.0100 & 8.29581 & 50 & 5 & 0.0100 & 9.13507 \\
& & 0.0250 & 6.89706 & & & 0.0250 & 7.01403 \\
& & 0.0500 & 5.76003 & & & 0.0500 & 5.51212 \\
& 0.1000 & 4.55274 & & & 0.1000 & 4.25339 \\
\hline
\end{tabular}

Since researchers find it necessary to know the exact critical values of the $T$ statistic, we offer a program to calculate the exact critical values of the $T$ statistic for small sample sizes. However, for example, we may not evaluate the exact critical value of the $T$ statistic for the case of $n=m=20$. This is because we have to consider ${ }_{40} C_{20}=137,846,528,820$ combinations to calculate the exact critical value. Therefore it is necessary to approximate the distribution of the $T$ statistic. Then, we first suggest a gamma approximation to the distribution of the $T$ statistic as follows:

$$
\Gamma(\alpha, \beta) \quad \text { where } \quad \alpha=\frac{4}{\kappa_{2}} \text { and } \beta=\frac{\kappa_{2}}{2} .
$$

$\Gamma(\alpha, \beta)$ converges to a chi-square distribution with two degrees of freedom when $N \rightarrow \infty$ (Lepage et al., 1986).

It is difficult to derive the cumulant generating function (CGF) of the $T$ statistic; we need to approximate the $\mathrm{CGF}$ of the $T$ statistic. Herein, we introduce the approximation 
of Easton and Ronchetti (1986) to the CGF of the statistic as follows:

$$
K(x) \approx K_{1}(x)=\sum_{i=1}^{4} \frac{\kappa_{i} x^{i}}{i !} .
$$

Kakizawa and Taniguchi (1994) defined a correction of the $K_{1}(x)$ as follows:

$$
K(x) \approx K_{2}(x)=\kappa_{1} x+\frac{\left(1+\kappa_{2}\right) x^{2}}{2 !}+\frac{\kappa_{3} x^{3}}{3 !}+\frac{\kappa_{4} x^{4}}{4 !} .
$$

In addition, Easton and Ronchetti (1986) considered another approximation as

$$
K(x) \approx K_{3}(x)=\kappa_{1} x+\frac{\kappa_{2}}{2} x^{2}+\log \left(1+\frac{\kappa_{3}}{6} x^{3}+\frac{3 \kappa_{4}}{72} x^{4}+\frac{\kappa_{3}^{2}}{72} x^{6}\right) .
$$

Additionally, Wang (1992) proposed a simple modification of $K_{1}(x)$ as follows:

$$
K(x) \approx K_{4}(x ; p)=\kappa_{1} x+\frac{\kappa_{2}}{2 !} x^{2}+\left(\frac{\kappa_{3}}{3 !} x^{3}+\frac{\kappa_{4}}{4 !} x^{4}\right) \eta_{p}(x),
$$

where

$$
\eta_{p}(x)=\exp \left\{-\frac{\kappa_{2} p^{2} x^{2}}{2}\right\}
$$

Wang defined $p$ as follows:

$$
p=\max \left(\frac{1}{2}, \inf \left\{q \mid K_{4}^{(2)}(x ; q) \geq 0 \text { for all } T\right\}\right) .
$$

By the first four cumulants, we may easily estimate the $i$ th cumulant of the $T$ statistic for $i \geq 5$ as follows:

$$
\sum_{i=5}^{\infty} \frac{2^{i-1} 2(i-1) ! N^{3(i-1)+1} x^{i}}{(N-2)^{i}(N+1)^{i-1}(N+2)^{i-1} i !}+O\left(N^{-1}\right) .
$$

Then we add (1) to $K_{1}(x)$ and named $K_{5}(x)$ as follows:

$$
\begin{aligned}
K(x) \approx & K_{1}(x)+\sum_{i=5}^{\infty} \frac{2^{i-1} 2(i-1) ! N^{3(i-1)+1} x^{i}}{(N-2)^{i}(N+1)^{i-1}(N+2)^{i-1} i !}+O\left(N^{-1}\right) \\
\approx & K_{1}(x)+\sum_{i=5}^{\infty} \frac{2^{i} N^{3 i-2} x^{i}}{(N-2)^{i}(N+1)^{i-1}(N+2)^{i-1} i} \\
= & K_{1}(x)-\frac{1}{3(N-2)^{4} N^{2}(N+1)^{3}(N+2)^{3}}\left[2 N ^ { 3 } x \left\{3\left(N^{3}+N^{2}-4 N-4\right)^{3}\right.\right. \\
& \left.+3 N^{3}\left(N^{3}+N^{2}-4 N-4\right)^{2} x+4(N-2) N^{6}(N+1)(N+2) x^{2}+6 N^{9} x^{3}\right\} \\
& \left.+3\left(N^{3}+N^{2}-4 N-4\right)^{4} \log \left(1-\frac{2 N^{3} x}{N^{3}+N^{2}-4 N-4}\right)\right] \\
= & K_{5}(x) .
\end{aligned}
$$


We may consider replacing the $K_{1}(x)$ to $K_{2}(x)$ in $K_{5}(x)$ as follows:

$$
\begin{aligned}
K(x) \approx K_{6}(x)= & K_{2}(x)-\frac{1}{3(N-2)^{4} N^{2}(N+1)^{3}(N+2)^{3}}\left[2 N ^ { 3 } x \left\{3\left(N^{3}+N^{2}-4 N-4\right)^{3}\right.\right. \\
& \left.+3 N^{3}\left(N^{3}+N^{2}-4 N-4\right)^{2} x+4(N-2) N^{6}(N+1)(N+2) x^{2}+6 N^{9} x^{3}\right\} \\
& \left.+3\left(N^{3}+N^{2}-4 N-4\right)^{4} \log \left(1-\frac{2 N^{3} x}{N^{3}+N^{2}-4 N-4}\right)\right] .
\end{aligned}
$$

\section{Saddlepoint approximation}

In this section, we consider a saddlepoint approximation (Daniels; 1954, 1987) to the distribution of the $T$ statistic. To obtain the saddlepoint approximation, we evaluate the first four derivatives of the CGF of the $T$ statistic. To determine the saddlepoint approximation to $\operatorname{Pr}(T \geq v)$, we solve the saddlepoint equation, $K^{(1)}(x)=v$, and use the unique solution $(x=\hat{x})$ to calculate

$$
\hat{w}=\sqrt{2(\hat{x} v-K(\hat{x}))} \operatorname{sgn}(\hat{x}) \quad \text { and } \quad \hat{u}=\hat{x} \sqrt{K^{(2)}(\hat{x})},
$$

where $\operatorname{sgn}(\hat{x})= \pm 1,0$ if $\hat{x}$ is positive, negative or zero and $K^{(j)}(\cdot)$ denotes the $j$ th derivative of $K(\cdot)$. The saddlepoint approximations to the cumulative distribution function of the $T$ statistic by using the normal-based Lugannani and Rice (L-R) formula for the tail probability is given by

$$
\operatorname{Pr}_{1}(T \geq v)=1-\Phi(\hat{w})+\phi(\hat{w})\left\{\frac{1}{\hat{u}}-\frac{1}{\hat{w}}\right\}
$$

and

$$
\operatorname{Pr}_{2}(T \geq v)=1-\Phi(\hat{w})+\phi(\hat{w}) S_{1},
$$

where $\phi(\cdot)$ is the standard Normal density function and $\Phi(\cdot)$ is the corresponding cumulative distribution function and

$$
S_{1}=\frac{1}{\hat{u}}-\frac{1}{\hat{w}}+\frac{1}{\hat{u}}\left(\frac{\zeta_{4}}{8}-\frac{5 \zeta_{3}^{2}}{24}\right)-\frac{\zeta_{3}}{2 \hat{u}^{2}}-\frac{1}{\hat{u}^{3}}+\frac{1}{\hat{w}^{3}} \quad \text { and } \quad \zeta_{r}=\frac{K^{(r)}(\hat{x})}{\left\{K^{(2)}(\hat{x})\right\}^{r / 2}} .
$$

Wood et al. (1993) generalized the L-R formula to a nonnormal-based saddlepoint approximation. Suppose that $G(w)$ is the CGF of the base distribution. For each $\xi$, let $w_{\xi}$ be the unique solution to the saddlepoint equation

$$
G^{(1)}\left(w_{\xi}\right)=\xi
$$

where $G^{(j)}(\cdot)$ denotes the $j$ th derivative of $G(\cdot)$. Then we find $\hat{\xi}$ to satisfy

$$
G\left(w_{\xi}\right)-\xi w_{\xi}=K^{(1)}(\hat{x})-v \hat{x},
$$

where $\hat{x}$ is obtained from $K^{(1)}(\hat{x})=v$.

Suppose now that $\Gamma$ and $\gamma$ are the cumulative distribution function and density of the distribution whose CGF is $G(w)$. Then we have the generalized L-R formula as follows:

$$
\operatorname{Pr}_{3}(T \geq v)=1-\Gamma(\hat{\xi})+\gamma(\hat{\xi})\left\{\frac{1}{\hat{u}_{\hat{\xi}}}-\frac{1}{w_{\hat{\xi}}}\right\}
$$


and

$$
\operatorname{Pr}_{4}(T \geq v)=1-\Gamma(\hat{\xi})+\frac{\gamma(\hat{\xi})}{\delta_{\hat{\xi}}}\left\{\frac{1}{\hat{u}_{\hat{\xi}}}-\frac{1}{w_{\hat{\xi}}}+S_{2}\right\}
$$

where

$$
\hat{u}_{\hat{\xi}}=\hat{u} \sqrt{G^{(2)}\left(w_{\hat{\xi}}\right)}, \quad \tau_{r}=\frac{G^{(r)}(\hat{\xi})}{\left\{G^{(2)}(\hat{\xi})\right\}^{r / 2}}, \quad \delta_{\hat{\xi}}=1+\frac{1}{8} \tau_{4}-\frac{5}{24} \tau_{3}^{2},
$$

and

$$
\begin{aligned}
S_{2}= & \frac{1}{8} *\left(\frac{\zeta_{4}}{\hat{u}_{\hat{\xi}}}-\frac{\tau_{4}}{w_{\hat{\xi}}}\right)-\frac{5}{24} *\left(\frac{\zeta_{3}^{2}}{\hat{u}_{\hat{\xi}}}-\frac{\tau_{3}^{2}}{w_{\hat{\xi}}}\right)-\frac{1}{2 G^{(2)}(\hat{\xi})^{\frac{1}{2}}} *\left(\frac{\zeta_{3}}{\hat{u}_{\hat{\xi}}^{2}}-\frac{\tau_{3}}{w_{\hat{\xi}}^{2}}\right) \\
& -\frac{1}{G^{(2)}(\hat{\xi})} *\left(\frac{1}{\hat{u}_{\hat{\xi}}^{3}}-\frac{1}{w_{\hat{\xi}}^{3}}\right) .
\end{aligned}
$$

\section{Numerical comparison}

In this section, we report on the accuracy of various approximations. We use the gamma, normal-based saddlepoint and nonnormal-based saddlepoint approximations. In the tables, the critical values were estimated by a simulation study which was $10,000,000$ times repetition. We list the upper tail probability of the proposed gamma approximation, namely $A_{G}$, and the limiting distribution, namely $A_{C}$ for each critical value of the $T$ statistic. In addition, $S A_{N L}$ and $S A_{N H}$ denote the normal-based saddlepoint approximations corresponding to $\operatorname{Pr}_{1}(\cdot)$ and $\operatorname{Pr}_{2}(\cdot)$, respectively. For a nonnormal-based saddlepoint approximation, specifically, we choose the chi-square distribution with one and two degrees of freedom in this paper because the limiting distribution is the chi-square distribution. The CGF of the chi-square distribution is

$$
G\left(w_{\hat{\xi}}\right)=-\frac{\nu}{2} \log \left(1-2 w_{\hat{\xi}}\right),
$$

where $\nu$ denotes the degrees of freedom. In the table of numerical results, $S A_{C L \nu}$ and $S A_{C H \nu}$ denote the chi-square-based saddlepoint approximations for $\operatorname{Pr}_{3}(\cdot)$ and $\operatorname{Pr}_{4}(\cdot)$, respectively.

Using an extra term approximation doesn't necessarily guarantee an improvement for any particular fixed finite sample sizes. Sometimes, it is possible for the corrections from the extra terms to worsen the approximation. Then, in this paper, we compared the numerical results of the saddlepoint approximations based on $K_{1}(x) K_{2}(x), K_{5}(x)$ and $K_{6}(x)$ for equal and unequal sample sizes. Since the saddlepoint approximation based on $K_{3}(x)$ and $K_{4}(x ; p)$ appeared to give a poorer approximation in the examples considered than that based on $K_{1}(x)$, we will not include this approach in the numerical examples presented in this section.

The results of Table 2 show that the saddlepoint approximation $S A_{N H}$ based on $K_{6}(x)$ is more accurate than the other saddlepoint approximations in upper tail probability. In addition, the $A_{G}$ approximation is also efficient to approximate the upper tail probability. The $A_{G}$ and $S A_{N H}$ based on $K_{5}(x)$ approximations are more satisfactory than the $A_{C}$ approximation for equal sample sizes. 
Approximations to the Nonparametric Statistic

Table 2: Tail probability for $n=m=30$

\begin{tabular}{llllll}
\hline & & 4.583 & 5.897 & 7.189 & 8.844 \\
\cline { 2 - 5 } & Simulation & 0.100 & 0.050 & 0.025 & 0.010 \\
& $A_{C}$ & 0.102 & 0.052 & 0.027 & 0.012 \\
& $A_{G}$ & 0.098 & 0.049 & 0.025 & 0.010 \\
\hline$K_{1}(x)$ & $S A_{N L}$ & 0.115 & 0.056 & 0.026 & 0.009 \\
& $S A_{N H}$ & 0.117 & 0.057 & 0.026 & 0.009 \\
& $S A_{C L 1}$ & 0.109 & 0.052 & 0.024 & 0.008 \\
& $S A_{C H 1}$ & 0.120 & 0.055 & 0.025 & 0.008 \\
& $S A_{C L 2}$ & 0.113 & 0.054 & 0.025 & 0.008 \\
& $S A_{C H 2}$ & 0.102 & 0.050 & 0.023 & 0.008 \\
\hline$K_{2}(x)$ & $S A_{N L}$ & 0.129 & 0.064 & 0.031 & 0.011 \\
& $S A_{N H}$ & 0.130 & 0.065 & 0.031 & 0.011 \\
& $S A_{C L 1}$ & 0.122 & 0.060 & 0.028 & 0.010 \\
& $S A_{C H 1}$ & 0.137 & 0.065 & 0.030 & 0.011 \\
& $S A_{C L 2}$ & 0.127 & 0.063 & 0.030 & 0.010 \\
& $S A_{C H 2}$ & 0.122 & 0.061 & 0.029 & 0.010 \\
\hline$K_{5}(x)$ & $S A_{N L}$ & 0.092 & 0.047 & 0.024 & 0.010 \\
& $S A_{N H}$ & 0.087 & 0.045 & 0.023 & 0.010 \\
& $S A_{C L 1}$ & 0.090 & 0.045 & 0.023 & 0.010 \\
& $S A_{C H 1}$ & 0.090 & 0.047 & 0.025 & 0.011 \\
& $S A_{C L 2}$ & 0.092 & 0.047 & 0.024 & 0.010 \\
& $S A_{C H 2}$ & 0.088 & 0.045 & 0.023 & 0.010 \\
\hline$K_{6}(x)$ & $S A_{N L}$ & 0.106 & 0.054 & 0.028 & 0.012 \\
& $S A_{N H}$ & 0.101 & 0.051 & 0.026 & 0.011 \\
& $S A_{C L 1}$ & 0.103 & 0.052 & 0.026 & 0.011 \\
& $S A_{C H 1}$ & 0.093 & 0.052 & 0.028 & 0.012 \\
& $S A_{C L 2}$ & 0.106 & 0.053 & 0.027 & 0.012 \\
& $S A_{C H 2}$ & 0.103 & 0.052 & 0.027 & 0.011 \\
\hline & & & & &
\end{tabular}

The results of Table 3 reveal that the $S A_{C H 2}$ based on $K_{1}(x)$ and $S A_{N H}$ based on $K_{6}(x)$ are more accurate than the other saddlepoint approximations. In addition, both of the saddlepoint approximations are more accurate and satisfactory than the $A_{C}$ approximation. However, the $A_{G}$ approximation was more suitable than the saddlepoint approximations for upper tail probability for unequal sample sizes. Therefore, the $S A_{N H}$ based on $K_{6}(x)$ and $A_{G}$ were efficient for approximating the distribution of the $T$ statistic.

\section{Conclusion}

In this paper, we consider a gamma approximation and various saddlepoint approximations to the distribution of the Lepage-type test which is a combination of the Wilcoxon and Mood statistic, named $T$. The first four cumulants of the $T$ statistic are derived. By the numerical results, the precision of the gamma approximation and $S A_{N H}$ based on $K_{6}(x)$ are shown to be superior to that of other normal-based and nonnormal-based saddlepoint approximations to the distribution of the Lepage-type test. In any case, the $A_{G}$ approximation is more accurate than the $A_{C}$ approximation. In addition, the approximation $A_{G}$ was more efficient than the $S A_{N H}$ based $K_{6}(x)$ approximation for equal and unequal sample 


\section{MURAKAMI}

Table 3: Tail probability for $n=40, m=20$

\begin{tabular}{llllll}
\hline & & 4.571 & 5.877 & 7.161 & 8.829 \\
\cline { 2 - 5 } & Simulation & 0.100 & 0.050 & 0.025 & 0.010 \\
& $A_{C}$ & 0.102 & 0.053 & 0.028 & 0.012 \\
& $A_{G}$ & 0.098 & 0.050 & 0.025 & 0.011 \\
\hline$K_{1}(x)$ & $S A_{N L}$ & 0.116 & 0.057 & 0.027 & 0.009 \\
& $S A_{N H}$ & 0.117 & 0.058 & 0.027 & 0.009 \\
& $S A_{C L 1}$ & 0.110 & 0.053 & 0.024 & 0.008 \\
$S A_{C H 1}$ & 0.121 & 0.056 & 0.026 & 0.009 \\
& $S A_{C L 2}$ & 0.114 & 0.055 & 0.026 & 0.009 \\
& $S A_{C H 2}$ & 0.103 & 0.051 & 0.024 & 0.008 \\
\hline$K_{2}(x)$ & $S A_{N L}$ & 0.130 & 0.066 & 0.031 & 0.011 \\
& $S A_{N H}$ & 0.130 & 0.066 & 0.032 & 0.011 \\
& $S A_{C L 1}$ & 0.122 & 0.061 & 0.029 & 0.010 \\
& $S A_{C H 1}$ & 0.139 & 0.067 & 0.031 & 0.011 \\
& $S A_{C L 2}$ & 0.127 & 0.064 & 0.030 & 0.011 \\
& $S A_{C H 2}$ & 0.122 & 0.061 & 0.029 & 0.010 \\
\hline$K_{5}(x)$ & $S A_{N L}$ & 0.093 & 0.048 & 0.025 & 0.011 \\
& $S A_{N H}$ & 0.088 & 0.046 & 0.024 & 0.010 \\
& $S A_{C L 1}$ & 0.091 & 0.046 & 0.024 & 0.010 \\
& $S A_{C H 1}$ & 0.092 & 0.048 & 0.025 & 0.011 \\
& $S A_{C L 2}$ & 0.093 & 0.047 & 0.025 & 0.011 \\
& $S A_{C H 2}$ & 0.089 & 0.046 & 0.024 & 0.010 \\
\hline$K_{6}(x)$ & $S A_{N L}$ & 0.107 & 0.055 & 0.028 & 0.012 \\
& $S A_{N H}$ & 0.102 & 0.052 & 0.027 & 0.012 \\
& $S A_{C L 1}$ & 0.104 & 0.053 & 0.027 & 0.012 \\
& $S A_{C H 1}$ & 0.095 & 0.053 & 0.028 & 0.012 \\
& $S A_{C L 2}$ & 0.107 & 0.053 & 0.028 & 0.012 \\
& $S A_{C H 2}$ & 0.104 & 0.053 & 0.027 & 0.012 \\
\hline & & & &
\end{tabular}

sizes, especially unequal sample sizes. Therefore, the $A_{G}$ statistic was suitable to estimate the critical value of the $T$ statistic.

\section{Appendix First four cumulants of the $T$ statistic}

First cumulant of the $T$ statistic:

$$
\kappa_{1}=2
$$

Second cumulant of the $T$ statistic:

$$
\kappa_{2}=\frac{4\left(A_{1}+A_{2}\right)}{35 n m(N-2)^{2}(N+1)(N+2)},
$$

where

$$
\begin{aligned}
& A_{1}=n m\left(35 N^{4}-127 N^{3}-626 N^{2}+1588 N+5184\right), \\
& A_{2}=-4 N(N+1)\left(2 N^{3}-29 N^{2}+37 N+251\right) .
\end{aligned}
$$


Third cumulant of the $T$ statistic:

$$
\kappa_{3}=\frac{16\left(A_{3}+A_{4}+A_{5}+A_{6}\right)}{35035 n^{2} m^{2}(N-2)^{3}(N+1)^{2}(N+2)^{2}},
$$

where

$$
\begin{aligned}
A_{3}= & n^{3}(N+m)\left(-35035 N^{7}+498212 N^{6}+2563853 N^{5}-17007490 N^{4}\right. \\
& \left.-81801784 N^{3}+181994024 N^{2}+1340455728 N+1740082176\right), \\
A_{4}= & n^{2} N\left(35035 N^{8}-529672 N^{7}-3470117 N^{6}+17886274 N^{5}+114223564 N^{4}\right. \\
& \left.-154233296 N^{3}-1803614736 N^{2}-3209149192 N-1331007552\right), \\
A_{5}= & 4 n N^{2}\left(7865 N^{7}+226566 N^{6}-219696 N^{5}-8105445 N^{4}-6940182 N^{3}\right. \\
& \left.+115789752 N^{2}+367266754 N+332751888\right), \\
A_{6}= & -6 N\left(7523 N^{8}+58206 N^{7}-348346 N^{6}-2060124 N^{5}+3905103 N^{4}\right. \\
& \left.+35872734 N^{3}+60357736 N^{2}+19827864 N-10223336\right) .
\end{aligned}
$$

Fourth cumulant of the $T$ statistic:

$$
\kappa_{4}=\frac{96\left(A_{7}+A_{8}+A_{9}+A_{10}+A_{11}+A_{12}\right)}{2977975 n^{3} m^{3}(N-2)^{4}(N+1)^{3}(N+2)^{3}},
$$

where

$$
\begin{aligned}
A_{7}= & n^{5}(2 N+m)\left(2977975 N^{10}-119447185 N^{9}-416453777 N^{8}\right. \\
& +9662189097 N^{7}+36504126558 N^{6}-348955046364 N^{5} \\
& -2043389380984 N^{4}+3044326120816 N^{3}+48316473341600 N^{2} \\
& +133264055195712 N+122585067270144) \\
A_{8}= & n^{3} N^{2}\left(2977975 N^{11}-149008145 N^{10}-1058925665 N^{9}+12716457417 N^{8}\right. \\
& +91920338798 N^{7}-415906988748 N^{6}-4510464677176 N^{5} \\
& -2171634664576 N^{4}+89662808498144 N^{3}+383917313837760 N^{2} \\
& +651986874923008 N+410763198283776), \\
A_{9}= & -n^{4} N\left(8933925 N^{11}-373122035 N^{10}-1570597275 N^{9}+30513701451 N^{8}\right. \\
& +137220485794 N^{7}-1080341110284 N^{6}-7363705791048 N^{5} \\
& +6524997969752 N^{4}+165622587603072 N^{3}+525118794908160 N^{2} \\
& +632456105636864 N+205381599141888), \\
A_{10}= & -8 n N^{2}\left(4512684 N^{11}+26145374 N^{10}-469694095 N^{9}-2464865903 N^{8}\right. \\
& +19034565943 N^{7}+144838776267 N^{6}-85995851346 N^{5} \\
& -3470780397296 N^{4}-12770870514146 N^{3}-18874143095350 N^{2} \\
& -7710625009828 N+4397374185240), \\
A_{11}= & 120 N\left(158220 N^{12}+70674 N^{11}-15606667 N^{10}-16323560 N^{9}\right. \\
& +702834509 N^{8}+2370214388 N^{7}-10772853205 N^{6}-84809116296 N^{5} \\
& -199114230869 N^{4}-155586112366 N^{3}+51248243068 N^{2} \\
& +55123084552 N-24966727664),
\end{aligned}
$$




$$
\begin{aligned}
A_{12}= & 8 n^{2} N\left(1847560 N^{12}+44667177 N^{11}-164746396 N^{10}-3933207360 N^{9}\right. \\
& +1719630496 N^{8}+173226771955 N^{7}+470836325354 N^{6} \\
& -2670141798630 N^{5}-19136609062424 N^{4}-45858483492450 N^{3} \\
& \left.-44546842988086 N^{2}-7710625009828 N+4397374185240\right) .
\end{aligned}
$$

\section{REFERENCES}

Anderson, T. W. and Darling, D. A. (1952). Asymptotic theory of certain goodness-of-fit criteria based on stochastic processes. The Annals of Mathematical Statistics 23, 193212.

Anderson, T. W. and Darling, D. A. (1954). A test of goodness of fit. Journal of the American Statistical Association 49, 765-769.

Ansari, A. R. and Bradley, R. A. (1960). Rank sum tests for dispersion. The Annals of Mathematical Statistics 31, 1174-1189.

Bean, R., Froda, S. and van Eeden, C. (2004). The normal, Edgeworth, saddlepoint and uniform approximations to the Wilcoxon-Mann-Whitney null-distribution: A numerical comparison. Journal of Nonparametric Statistics 16, 279-288.

Büning, H. (2002). Robustness and power of modified Lepage, Kolmogorov-Smirnov and Cramér-Von Mises two-sample tests. Journal of Applied Statistics 29, 907-924.

Büning, H. and Thadewald, T. (2000). An adaptive two-sample location-scale test of Lepage type for symmetric distributions. Journal of Statistical Computation and Simulation $\mathbf{6 5}$, $287-310$.

Butler, R. W. (2007). Saddlepoint approximations with applications. Cambridge University Press.

Chen, Q. and Giles, D. E. (2008). General saddlepoint approximations: Application to the Anderson-Darling test statistic. Communications in Statistics : Simulation and Computation 37, 789-804.

Daniels, H. E. (1954). Saddlepoint approximations in statistics. The Annals of Mathematical Statistics 25, 631-650.

Daniels, H. E. (1987). Tail Probability Approximations. International Statistical Review 55, $37-48$.

Easton, G. S. and Ronchetti, E. (1986). General saddlepoint approximations with applications to $L$ statistics. Journal of the American Statistical Association 81, 420-430.

Froda, S. and van Eeden, C. (2000). A uniform saddlepoint expansion for the nulldistribution of the Wilcoxon-Mann-Whitney statistic. The Canadian Journal of Statistics 28, 137-149.

Gibbons, J. D. and Chakraborti, S. (2003). Nonparametric statistical Inference, 4th Edition. Dekker.

Giles, D. E. (2001). A saddlepoint approximation to the distribution function of the Anderson-Darling test statistic. Communications in Statistics - Simulation and Computation 30, 899-905.

Goutis, C. and Casella, G. (1999). Explaining the saddlepoint approximation. The American Statistician 53, 216-224.

Huzurbazar, S. (1999). Practical saddlepoint approximations. The American Statistician 5, $225-232$.

Jensen, J. L. (1995). Saddlepoint Approximations. New York: Oxford University Press. 
Kolassa, J. E. (2006). Series Approximation Methods in Statistics. New York: SpringerVerlag.

Lepage, Y. (1971). A combination of Wilcoxon's and Ansari-Bradley's statistics. Biometrika $\mathbf{5 8}, 213-217$.

Lepage, Y., Houde, L. and Cloutier, R. (1986). La loi d'une statistique combinant les statistiques de Wilcoxon et d'Andari-Bradley. Annales des sciences mathématiques du Québec 10, 189-197.

Lugannani, R. and Rice, S. O. (1980). Saddlepoint approximation for the distribution of the sum of independent random variables. Advances in Applied Probability 12, 475-490.

Kakizawa, Y. and Taniguchi, M. (1994). Higher order asymptotic relation between edgeworth approximation and saddlepoint approximation. Journal of the Japan Statistical Society 24, 109-119.

Mood, A. M. (1954). On the asymptotic efficiency of certain nonparametric two-sample tests. The Annals of Mathematical Statistics 25, 514-522.

Murakami, H. (2007). Lepage type statistic based on the modified Baumgartner statistic. Computational Statistics and Data Analysis 51, 5061-5067.

Murakami, H. (2009). Saddlepoint Approximations to the Limiting Distribution of the Modified Anderson-Darling Test Statistic. Communications in Statistics - Simulation and Computation 38, 2214-2219.

Murakami, H. and Kamakura, T. (2009). A saddlepoint approximation to the distribution of Jonckheere-Terpstra test. Journal of the Japan Statistical Society 39, 143-153.

Murakami, H. (2010). A numerical comparison of the normal and some saddlepoint approximations to a distribution-free test for stochastic ordering in the competing risks model. Computational Statistics 25, 633-643.

Neuhäuser, M. (2000). An exact two-sample test based on the Baumgartner-Weiss-Schindler statistic and a modification of Lepage's test. Communications in Statistics : Theory and Methods 29, 67-78.

Pettitt, A. N. (1976). A two-sample Anderson-Darling rank statistic. Biometrika 63, 161168.

Reid, N. (1988). Saddlepoint methods and statistical inference (with discussion). Statistical Science 3, 213-238.

Sinclair, C. D. and Spurr, B. D. (1988). Approximations to the distribution function of the Anderson-Darling test statistic. Journal of the American Statistical Association 83, $1190-1191$.

Sinclair, C. D., Spurr, B. D. and Ahmad, M. I. (1990). Modified Anderson-Darling test. Communications in Statistics : Theory and Methods 19, 3677-3686.

Wang, S. (1992). General saddlepoint approximations in the bootstrap. Statistics and Probability Letters 13, 61-66.

Wood, A. T. A., Booth, J. G. and Butler, R. W. (1993). Saddlepoint approximations to the CDF of some statistics with nonnormal limit distributions. Journal of the American Statistical Association 88, 680-686.

(Received: July 26, 2010, Accepted: December 6, 2010) 
